Théorie des groupes/Group Theory

\title{
An invariant for subfactors in the von Neumann algebra of a free group
}

\section{Florin RĂDULESCU}

\begin{abstract}
In this Note we are considering a new invariant for subfactors in the von Neumann algebra $\mathscr{L}\left(\mathrm{F}_{k}\right)$ of a free group. This invariant is obtained by computing the Connes's $\chi$ invariant for the enveloping von Neumann algebra in the iteration of the Jone's basic construction for the given inclusion. In the case of the subfactors considered in [22], [24] this invariant is easily computed as a relative $\chi$ invariant, in the form considered in [14].

One considers the inclusion $\mathscr{L}\left(\mathrm{F}_{n}\right) \subseteq \mathscr{A}=\mathscr{L}\left(\mathrm{F}_{n}\right) \searrow_{\theta} \mathbb{Z}_{k}{ }^{2}$, where $\theta$ is an injective homomorphism from $\mathbb{Z}_{k}$ into Out $\left(\mathscr{L}\left(\mathrm{F}_{n}\right)\right.$ ) (i.e. a $\left.\mathbb{Z}_{n}{ }^{-k e r n e l}\right)$ with minimal period $k^{2}$ [in Aut $\left(\mathscr{L}\left(\mathrm{F}_{n}\right)\right)$ ]. Then there

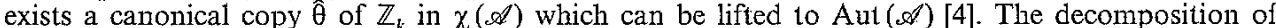
the generator of the dual action of $\mathbb{Z}_{k}$ on $\mathscr{A} \times \mathbb{A}_{\hat{\theta}} \mathbb{Z}_{k}$ as the product of a centrally trivial automorphism and an almost inner automorphism, gives an action of $\mathbb{Z}_{k}{ }^{2} \oplus \mathbb{Z}_{k}{ }^{2}$ on the algebra $\mathscr{A} \rtimes_{\hat{\theta}} \mathbb{Z}_{k}$. The algebraic invariants [9] for this last action give a more subtle invariant for $\theta$.

As an application we show that, contrary to the case of finite group actions (or more general Gkernels) on the hyperfine $\mathrm{II}_{1}$ factor (settled in [2], [9], [18]), there exists non outer conjugate, injective homomorphisms (i.e. two $\mathbb{Z}_{2}$-kernels) from $\mathbb{Z}_{2}$ into Out $\left(\mathscr{L}\left(\mathrm{F}_{k}\right)\right.$ ), with non-trivial obstruction to lifting to an action on $\mathscr{L}\left(\mathrm{F}_{k}\right)$. Moreover the algebraic invariants [3] do not distinguish between these two $\mathbb{Z}_{2}$-kernels. Also, there exists two non-outer conjugate, outer actions of $\mathbb{Z}_{2}$ on $\mathscr{L}\left(\mathrm{F}_{k}\right) \otimes \mathrm{R}$ that are neither almost inner or centrally trivial.
\end{abstract}

Un invariant pour les sous-facteurs de l'algèbre de von Neumann associée à un groupe libre

Résumé - Nous introduisons un nouvel invariant pour les sous-facteurs de l'algèbre de von Neumann $\mathscr{L}\left(\mathrm{F}_{k}\right)$ d'un groupe libre $\mathrm{F}_{k}$, en calculant l'invariant $\chi$ de Connes pour l'algèbre enveloppante de la construction de base de Jones associée à l'inclusion donnée. Dans le cas des sous-facteurs considérés dans [21], [24] cet invariant peut être facilement calculé en utilisant une version relative pour $\chi$, considérée dans [14].

On considère une inclusion de type $\mathscr{L}\left(\mathrm{F}_{n}\right) \subseteq \mathscr{A}=\mathscr{L}\left(\mathrm{F}_{n}\right) \times_{\theta} \mathbb{K}_{t} 2$, où $\theta$ est un homomorphisme injectif de $\mathbb{Z}_{k}$ dans $\operatorname{Out}\left(\mathscr{L}\left(\mathrm{F}_{n}\right)\right)$, de période minimale $k^{2}$ [dans Aut $\left.\left(\mathscr{L}\left(\mathrm{F}_{n}\right)\right)\right]$. Alors il existe un sous-groupe canonique $\mathbb{Z}_{k} \subseteq$ Aut $(\mathscr{A})$ dans $\chi(\mathscr{A})$ [4]. La décomposition du générateur de l'action duale de $\mathbb{Z}_{k}$ sur $\mathscr{A} \times \mathbb{Z}_{k}$, comme le produit d'un automorphisme centralement trivial et d'un automorphisme dans Int $(\mathscr{A})$, donne une action de $\mathbb{Z}_{k}{ }^{2} \oplus \mathbb{Z}_{k}{ }^{2}$ sur $\mathscr{A} \rtimes \mathbb{Z}_{k}$. On obtient ainsi un invariant plus subtil pour $\theta$, en considérant les invariants algébrique [9] de l'action de $\mathbb{Z}_{k}{ }^{2} \oplus \mathbb{Z}_{k}{ }^{2}$.

Comme application nous montrons que, contrairement au cas du facteur hyperfini de type $\mathrm{II}_{1}$ (analysé dans [2], [3], [9], [18]), il existe deux homomorphismes injectifs de $\mathbb{Z}_{2}$ dans $\operatorname{Out}\left(\mathscr{L}\left(\mathrm{F}_{k}\right)\right)=\operatorname{Aut}\left(\mathscr{L}\left(\mathrm{F}_{k}\right)\right) / \operatorname{Int}\left(\mathscr{L}\left(\mathrm{F}_{k}\right)\right)$, qui ont les mênes invariants algébrique [3], mais qui ne sont pas extérieurement conjuguées.

Version française abrégée - Le but de cette Note est d'introduire un nouvel invariant pour les sous-facteurs des facteurs de type $\mathrm{II}_{1}$ associés à un groupe libre. L'invariant que nous proposons est obtenu en calculant l'invariant $\chi$ de Connes [4] pour l'algèbre de von Neumann enveloppante de la construction de base de Jones, pour l'inclusion donnée. L'invariant $\chi$ a été introduit par $A$. Connes $([4],[5])$ pour résoudre un problème très difficile posé par Sakai [27] concernant l'existence d'un facteur de type $\mathrm{II}_{1}$ non anti-isomorphe à luimême.

On rappelle que si $M$ est un facteur de type $\mathrm{II}_{1}$, alors l'invariant $\chi(M)$ est le groupe (commutatif, voir [4]) $\chi(M)=(\mathrm{Ct}(\mathrm{M}) \cap \overline{\operatorname{Int}(M))} / \operatorname{Int}(\mathrm{M}) \leqq$ Out $(\mathrm{M})$ où Aut $(\mathrm{M})$ est le groupe d'automorphismes de $M, \operatorname{Int}(M)$ est le groupe des automorphismes intérieurs et $\operatorname{Ct}(M)$ est défini par

$$
\operatorname{Ct}(M)=\left\{\alpha \in \operatorname{Aut}(M),\left\|\alpha\left(x_{n}\right)-x_{n}\right\|_{2} \rightarrow 0, \text { pour toute suite centralisante } x_{n}\right\} .
$$

Note présentée par Alain ConNes. 
L'INVARIANT. - Soit $\mathrm{A} \subseteq \mathrm{B}$ une inclusion d'algèbres de von Neumann finies, soit $\mathrm{E}_{\mathrm{A}}^{\mathrm{B}}: \mathrm{B} \rightarrow \mathrm{A}$ une espérance conditionnelle de $\mathrm{B}$ dans $\mathrm{A}$ par rapport à une trace fidèle, normale sur $\mathrm{B}$ (invariante par $\mathrm{E}$ ) et soit $\mathscr{A}_{\infty}$ l'algèbre enveloppante de l'inclusion $\mathrm{A} \subseteq \mathrm{B}$ i. e. le terme à l'infini dans l'itération de la construction de base de fones [7] pour l'inclusion donnée). Dans ces conditions,

$$
\chi\left(\mathscr{A}_{\infty}\right) \subseteq \operatorname{Out}\left(\mathscr{A}_{\infty}\right),
$$

est un invariant pour l'inclusion $\mathrm{A} \subseteq \mathrm{B}$. (Comme dans [4], la force de l'invariant vient du fait que l'on considère $\chi\left(\mathscr{A}_{\infty}\right)$, non seulement comme un groupe abstrait, mais comme un sousgroupe de Out $\left.\left(\mathscr{A}_{\infty}\right)\right)$.

Dans les exemples de sous-facteurs des algèbres de von Neumann des groupes libres considérés dans [24], on peut facilement montrer que cet invariant cö̈ncide avec l'invariant relatif $\chi[14]$, pour une inclusion des facteurs hyperfinis associés à l'inclusion donnée.

THÉORÈME. - Soit $\mathrm{N} \cong \mathrm{M}$ une inclusion d'algèbres finies, à prédual séparable et telle qu'il existe une base orthonormale de $\mathrm{M}$ sur $\mathrm{N}$, de type Pimsner et Popa [20], pour une espérance conditionnelle de $\mathrm{M}$ sur $\mathrm{N}$. Soit $\mathscr{L}\left(\mathrm{F}_{k}\right)$ le facteur de type $\mathrm{II}_{1}$ associé à un groupe libre $\mathrm{F}_{k}$, $k \geqq 2$. Si $\mathscr{A}=\left(\mathscr{L}\left(\mathrm{F}_{k}\right) *_{\mathrm{N}} \mathrm{M}\right.$ est le produit libre, amalgamé réduit des algèbres de von Neumann introduit dans [22], alors

$$
\chi(\mathscr{A}) \cong \chi(\mathrm{N}, \mathrm{M}) .
$$

Ici $\chi(\mathrm{N}, \mathrm{M})$ est l'invariant relatif de Connes pour l'inclusion $\mathrm{N} \subseteq \mathrm{M}$, considéré dans [14].

Comme application des résultats précédents, nous allons construire deux homomorphismes injectifs de $\mathbb{Z}_{2}$ dans $\operatorname{Out}\left(\mathscr{L}\left(\mathrm{F}_{k}\right)\right.$ ), non extérieurement conjugués, tels que tous les invariants algébriques (voir [9], [3], [18]) coïncident.

ThÉORÈme. - Pour tout $k \in \mathbb{Z}, k \geqq 2$, il existe deux automorphismes $\alpha_{i} \in \operatorname{Aut}\left(\mathscr{L}\left(\mathrm{F}_{k}\right)\right)$, tels que les images de $\alpha_{i}$ dans $\operatorname{Out}\left(\mathscr{L}\left(\mathrm{F}_{k}\right)\right)$ sont non conjugués et d'ordre deux. De plus, tous les invariants algébriques [3] pour les deux homomorphismes injectifs de $\mathbb{Z}_{2}$ dans $\operatorname{Out}\left(\mathscr{L}\left(\mathrm{F}_{k}\right)\right)$ coïncident.

Corollary. - Il existe des sous-facteurs de $\mathscr{L}\left(\mathrm{F}_{k}\right)$, de profondeur finie ([7], [17]), non conjugués et tels que tous les invariants des deux inclusions provenant des commutants relatifs dans la construction de base ([7], [18], [21]) sont identiques.

THE INVARIANT. - Given $\mathrm{A} \subseteq \mathrm{B}$ an inclusion of type $I_{1}$ factors, of finite index, we let $\mathrm{B}_{\infty}$ be the enveloping algebra for the tower of algebras in the (iterated) Jones's basic construction [7] for $\mathrm{A} \subseteq \mathrm{B}$. Then

$$
\chi\left(\mathrm{B}_{\infty}\right) \cong \text { Out }\left(\mathrm{B}_{\infty}\right)
$$

is a conjugacy invariant for $\mathrm{A} \subseteq \mathrm{B}$.

Here, for a type $\mathrm{II}_{1}$ factor $\mathrm{M}, \chi(\mathrm{M})$ denotes the Connes's invariant for $\mathrm{M}$ [4]. The $\chi(\mathrm{M})$ invariant was introduced by Connes [4] in connection to a breakthrough construction that showed the existence of a type $\mathrm{II}_{1}$ factor not antiisomorphic to itself.

Recall that $\chi(M)=(\mathrm{Ct}(\mathrm{M}) \cap \overline{\operatorname{Int}(\mathrm{M}))} / \operatorname{Int}(\mathrm{M})$. Here $\mathrm{Ct}(\mathrm{M})$ is the set of all automorphisms $\alpha$ of $M$ that have the property that $\left\|\alpha\left(x_{n}\right)-x_{n}\right\|_{2} \rightarrow 0$, whenever $x_{n}, n \in \mathbb{Z}$ is a central sequence in $M$ (i.e. a bounded sequence in $M$ with $\left\|\left[x_{n}, y\right]\right\|_{2} \rightarrow 0$ for any $y \in M$ ). $\overline{\operatorname{Int}(\mathrm{M})}$ is the closure of Int (M) in the pointwise convergence topology on $\mathrm{M}$, while Int $(M)$ is the set of inner automorphisms of $M$. 
We will show that for the subfactors in free group algebras, considered in [24] (or for those considered in [22]), this invariant may be computed and it coincides with the relative form of the invariant $\chi[14]$ for an associated pair of hyperfinite factors.

Using the full strength of the invariant (which comes as in [4] by considering $\chi\left(\mathrm{B}_{\infty}\right)$ as a subgroup in $\operatorname{Out}\left(\mathrm{B}_{\infty}\right)=\operatorname{Aut}\left(\mathrm{B}_{\infty}\right) / \operatorname{Int}\left(\mathrm{B}_{\infty}\right)$, rather than as an abstract abelian group (see also [8], [12], [27])) we obtain as an application that the classification theory for finite group actions (and for G-kernels) on type $\mathrm{II}_{1}$ factors such as $\mathscr{L}\left(\mathrm{F}_{k}\right)$ (the von Neumann algebra of a free group $\mathrm{F}_{k}$ ) is different from the corresponding classification theory on the hyperfinite $\mathrm{II}_{1}$ factor ([2], [3], [9], [18]).

THEOREM. - There exists two injective homomorphisms from $\mathbb{Z}_{2}$ into Out $\left(\mathscr{L}\left(\mathrm{F}_{k}\right)\right)$, for any $k \in \mathbb{Z}, k \geqq 2$, non-liftable to an action of $\mathbb{Z}_{2}$ on $\mathscr{L}\left(\mathrm{F}_{k}\right)$ [i.e. two $\mathbb{Z}_{2}$-kernels on $\mathscr{L}\left(\mathrm{F}_{k}\right)$ with non-trivial obstruction] that are not outer conjugate (in Out) and for which all the algebraic invariants ([2], [9]) coincide.

Explicitly this means that there exists two (non-inner) automorphisms $\alpha_{i}, i=1,2$ on $\mathscr{L}\left(\mathrm{F}_{k}\right)$, having non conjugate images (of order two) in $\operatorname{Out}\left(\mathscr{L}\left(\mathrm{F}_{k}\right)\right)$. Moreover; both automorphisms are not liftable to actions of $\mathbb{Z}_{2}$ on $\mathscr{L}\left(\mathrm{F}_{k}\right)\left(i . e \alpha_{i}^{2}=\operatorname{Ad} g_{i}, \alpha_{i}\left(g_{i}\right)=-g_{i}\right.$, $g_{i}=g_{i}^{+}-g_{i}^{-}$, with $g_{i}^{ \pm}$projections of trace $1 / 2$ in $\mathscr{L}\left(\mathrm{F}_{k}\right)($ see [3], [9])).

By [19], the actions on $\mathscr{L}\left(\mathrm{F}_{\infty}\right)$, obtained by multiplying the generators by complex numbers of modulus 1 are distinguished (modulo outer conjugacy) by the topology induced on $\mathbb{Z}$ from Aut $\mathscr{L}\left(\mathrm{F}_{\infty}\right)$. This is easily seen not to work in the case of finite cyclic groups. For example all $\mathbb{Z}_{2}$ actions on $\mathscr{L}\left(\mathrm{F}_{2}\right)$ that are obtained by multiplying the generators with \pm 1 or by switching the generators, are conjugate via elements in Aut $\mathscr{L}\left(\mathrm{F}_{2}\right)$, which belong to the image of the $\mathrm{O}(2)$ action on $\mathscr{L}\left(\mathrm{F}_{2}\right)$, considered in [29].

Corollary. - There exists two non-conjugate, finite depth subfactors in $\mathscr{L}\left(\mathrm{F}_{k}\right)$ having the same higher relative commutant invariants ([17], [7], [21]).

It is plausible that an invariant of the type considered in this Note could be used to settle Kadison's problem in [13] (see also Sakai's book [26], Problem 4.4.44) on the isomorphism class of the algebras associated to free groups.

We are indebted to D. Bisch, V. F. R. Jones, S. Popa and D. Voiculescu for useful comments on this Note.

For the proof of the next lemma see the proof of lemma 4.3.3 in [26].

LeMma 1. - Let $\mathrm{N} \cong \mathrm{M}$ be a pair of separable, finite von Neumann algebras so that there exists an orthonormal Pimsner-Popa [20] basis for the inclusion $\mathrm{N} \subseteq \mathrm{M}$, with respect to some faithful, normal conditional expectation from $\mathrm{M}$ onto $\mathrm{N}$. Assume that $\mathrm{N}, \mathrm{M}$ have centers with trivial intersection.

Then the von Neumann algebra reduced free product $\mathscr{A}=\left(\mathscr{L}\left(\mathrm{F}_{k}\right) \otimes \mathrm{N}\right) *_{\mathrm{N}} \mathrm{M}$ (which by [22] is a type $\mathrm{II}_{1}$ factor) has the property that $\left\|\mathrm{E}_{\mathrm{N}}^{\mathscr{A}}(\mathrm{y})-y\right\|_{2} \leqq 14 \max _{i=1,2}\left\|\left[g_{i}, y\right]\right\|_{2}$, with respect to the induced normal faithful conditional expectation $\mathrm{E}_{\mathrm{N}}^{\mathscr{s}}$ from $\mathscr{A}$ into $\mathrm{N}$. Here $g_{i}, i=1,2$ are two of the $k$ generators in $\mathrm{F}_{k}$, while $\|x\|^{2}=\tau\left(x^{*} x\right), x \in \mathscr{A}$ and $\tau$ is the trace on $\mathscr{A}$. In particular all central sequences in $\mathscr{A}$ are asymptotically contained in $\mathrm{N}$.

This lemma together with lemma 1.4 in [22] and lemma 3 in [11] gives:

THEOREM 2. - Let $\mathrm{N} \cong \mathrm{M}$ be a pair of separable, finite von Neumann algebras so that, with respect some faithful normal conditional expectation from $\mathrm{M}$ into $\mathrm{N}$, there exists an orthonormal Pimsner Popa [20] basis for the given inclusion. Assume that N, M have 
centers with trivial intersection. Let $\mathscr{A}=\left(\mathscr{L}\left(\mathrm{F}_{k}\right) \otimes \mathrm{N}\right) *_{\mathrm{N}} \mathrm{M}$. Then

$$
\chi\left(\left(\mathscr{L}\left(\mathrm{F}_{k}\right) \otimes \mathrm{N}\right) *_{\mathrm{N}} \mathrm{M}\right) \cong \chi(\mathrm{N}, \mathrm{M})
$$

Moreover in this identification, corresponding elements in $\chi(\mathscr{A})$ and $\chi(\mathrm{N}, \mathrm{M})$ have the same obstructions to lifting from the quotient.

Here $\chi(N, M)$ is the relative $\chi$ invariant [14] for the inclusion $\mathrm{N} \subseteq \mathrm{M}$, where $\mathrm{N}, \mathrm{M}$ of separable finite von Neumann algebras:

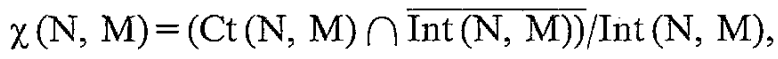

where $\operatorname{Ct}(\mathrm{N}, \mathrm{M})$ is the set of all automorphisms of $\mathrm{M}$, leaving $\mathrm{N}$ invariant and acting asymptotically trivial on central sequences for $M$ that are contained in $N$ and $\operatorname{Int}(N, M)$ is the set of all inner automorphisms of $\mathrm{M}$ that are implemented by unitaries in $\mathrm{N}$.

Corollary 3. - Let $\mathscr{C}=(\mathrm{A} \supseteqq \mathrm{B} \supseteqq \mathrm{C} ; \mathrm{A} \supseteqq \mathrm{C} \supseteqq \mathrm{D})$ be a commuting square [21] of finite dimensional algebras, which is irreducible (i. e. the centers of $\mathrm{A}, \mathrm{B}$ and respectively $\mathrm{C}, \mathrm{D}$ have trivial intersection) and $\lambda$-Markov ([7], [6]) i.e. there exists a $\lambda$-Markov trace, in the sense of Jones [7] for $\mathrm{C} \subseteq \mathrm{A}$, which restricts to a $\lambda$-Markov trace for $\mathrm{D} \subseteq \mathrm{B})$.

$\left.B y[24]\left(\mathscr{L}\left(\mathrm{F}_{k}\right) \otimes \mathrm{B}\right) *_{\mathrm{B}} \mathrm{A} \supseteqq\left(\mathscr{L}\left(\mathrm{F}_{k}\right)\right) \otimes \mathrm{D}\right) *_{\mathrm{D}} \mathrm{C}$ is a finite index inclusion of type $\mathrm{II}_{1}$ factors [that are isomorphic $\mathscr{L}\left(\mathrm{F}_{\mathrm{N}}\right)$ for some $\mathrm{N}>1$ ]. Let $\mathscr{A}_{\infty}$ be the envoloping von Neumann algebra for the tower of algebras in the iterated Jones's basic construction of the given inclusion. Note that $\mathscr{A}_{\infty}$ is isomorphic to $\left(\mathscr{L}\left(\mathrm{F}_{k}\right) \otimes \mathrm{B}_{\infty}\right) *_{\mathrm{B}_{\infty}} \mathrm{A}_{\infty}$. Here $\mathrm{B}_{\infty}, \mathrm{A}_{\infty}$ are obtained by iterating the basic construction for the inclusions $\mathrm{D} \subseteq \mathrm{B}$ and respectively $\mathrm{C} \subseteq \mathrm{A}$. Then

$$
\chi\left(\mathscr{A}_{\infty}\right)=\chi\left(\mathscr{L}\left(\mathrm{F}_{k}\right) \otimes \mathrm{B}_{\infty} * \mathrm{~B}_{\infty} \mathrm{A}_{\infty}\right) \cong \chi\left(\mathrm{B}_{\infty}, \mathrm{A}_{\infty}\right) .
$$

LemMa 4 ([17], [14], [15], [1], [23]). - Let $\theta$ be a $\mathbb{Z}_{4}$ action on a copy $\mathrm{R}_{-1}$ of the hyperfinite $\mathrm{II}_{1}$ factor so that $\theta$ induces a $\mathbb{Z}_{2}$-kemel on $\mathrm{R}_{-1}$ with obstruction -1 [3]. Let $\mathrm{R}_{0}=\mathrm{R}_{-1} \times_{\theta} \mathbb{Z}_{4}$. Then $\chi\left(\mathrm{R}_{-1}, \mathrm{R}_{0}\right) \cong \mathbb{Z}_{2} \oplus \mathbb{Z}_{2}$.

Moreover, for exactly one of the two copies of $\mathbb{Z}_{2}$ in $\chi\left(\mathrm{R}_{-1}, \mathrm{R}_{0}\right)$, there exists no obstruction to lifting from the quotient.

We may assume (see [10]) that

$$
\mathrm{R}_{-1}=\left\{g \mathrm{U}_{0}^{2}, \mathrm{U}_{1}, \mathrm{U}_{2}, \ldots\right\}^{\prime \prime} \leqq\left\{g, \mathrm{U}_{0}, \mathrm{U}_{1}, \ldots,\right\}^{\prime \prime}=\mathrm{R}_{0} .
$$

The $k$-th step in the iterated basic construction for this construction is:

$$
\left.\mathrm{R}_{k}=\left\{g \mathrm{U}_{-1}^{2} \ldots \mathrm{U}_{-(k-1)}^{2}, \mathrm{U}_{-k}, \mathrm{U}_{-(k+1)}, \ldots\right\}\right\}^{\prime \prime},
$$

where $\left(U_{k}\right)_{k \in \mathbb{Z}}$ is a family of unitaries with four point spectrum so that each spectral projection for $U_{k}$ has trace $1 / 4$ and $g=g_{+}-g_{-}$"is a selfadjoint unitary whose spectral projections $g_{ \pm}$have trace $1 / 2$. We have the following relations $\mathrm{U}_{k}^{4}=1, k \in \mathbb{Z}$, $\mathrm{U}_{k} g \mathrm{U}_{k}^{*}=-g$, if $k=0,1, \mathrm{U}_{k} g \mathrm{U}_{k}^{*}=g$, if $k \in \mathbb{Z} /\{0,1\}$ and $\mathrm{U}_{k} \mathrm{U}_{k+1} \mathrm{U}_{k}^{*}=\sqrt{-1} \mathrm{U}_{k+1}$ (the trace on this construction is specified by requiring that each non-trivial monomial in the $\mathrm{U}_{k}$ 's and $g$ have zero trace). With this notations, a commuting square for the inclusion $\mathrm{R}_{-1} \subseteq \mathrm{R}_{0}$ is given by

$$
\begin{gathered}
\mathrm{D}=\{g\}^{\prime \prime} \leqq \mathrm{C}=\left\{g \mathrm{U}_{-1}^{2}, \mathrm{U}_{-1}\right\}^{\prime \prime} ; \\
\mathrm{B}=\left\{g \mathrm{U}_{0}^{2}, \mathrm{U}_{0}\right\}^{\prime \prime} \cong \mathrm{A}=\left\{g \mathrm{U}_{0}^{2} \mathrm{U}_{-1}^{2}, \mathrm{U}_{-1}, \mathrm{U}_{0}\right\}^{\prime \prime} .
\end{gathered}
$$

Since $\mathrm{U}_{0} \in \mathrm{B}$ normalizes $\mathrm{A}$ and $\left.\mathrm{Ad} \mathrm{U}_{0}^{2}\right|_{\mathrm{C}}=\left.\mathrm{Ad} g\right|_{\mathrm{C}}$, while $g \in \mathrm{D}$, we get:

Lemma 5. - With $\mathrm{A}, \mathrm{B}, \mathrm{C}, \mathrm{D}$ as before, $\left(\mathscr{L}\left(\mathrm{F}_{k}\right) \otimes \mathrm{B}\right) *_{\mathrm{B}} \mathrm{A}$ is isomorphic to the cross product of $\left(\mathscr{L}\left(\mathrm{F}_{k}\right) \otimes \mathrm{D}\right) *_{\mathrm{D}} \mathrm{C}$ by the $\mathbb{Z}_{4}$ action on $\left(\mathscr{L}\left(\mathrm{F}_{k}\right) \otimes \mathrm{D}\right) *_{\mathrm{D}} \mathrm{C}$ induced by $\mathrm{Ad}_{0}$. [Note that this action is in fact an injective $\mathbb{Z}_{2}$-kernel on $\left(\mathscr{L}\left(\mathrm{F}_{k}\right) \otimes \mathrm{D}\right) *_{\mathrm{D}} \mathrm{C}$ with obstruction-1 to lifting (to Aut).] 
Moreover, by Corollary 3, the enveloping algebra for the inclusion before is $\mathscr{A}=\left(\mathscr{L}\left(\mathrm{F}_{k}\right) \otimes \mathrm{R}_{-1}\right) *_{\mathrm{R}_{-1}} \mathrm{R}_{0}$ and hence, by the preceding lemma, $\chi(\mathscr{A}) \cong \mathbb{Z}_{2} \oplus \mathbb{Z}_{2}$ is genera-

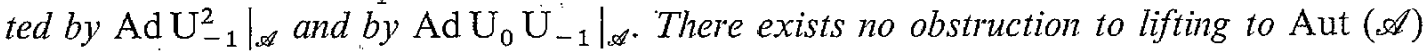
for the first copy of $\mathbb{Z}_{2}$, while for the second copy of $\mathbb{Z}_{2}$, the obstruction is -1 .

LemMA 6. - With the notations before, let $\mathscr{B}=\mathscr{A} \times \mathbb{Z}_{2}$, where the $\mathbb{Z}_{2}$ action on $\mathscr{A}$ is induced by $\left.\mathrm{Ad} \mathrm{U}_{1}^{2}\right|_{\mathscr{A}} \in \chi(\mathscr{A})$ (this action is an invariant for $\mathscr{A}$ ). Let $s$ be the dual action on the cross product. Then $\mathscr{B}$ isomorphic to $\left(\mathscr{L}\left(\mathrm{F}_{k}\right) \otimes \mathrm{Q}_{-1}\right) * \mathrm{Q}_{-1} \mathrm{Q}_{0}$, where $\mathrm{Q}_{-}=\left\{\mathrm{R}_{-1}, \mathrm{U}_{-1}^{2}\right\}^{\prime \prime}, \mathrm{Q}_{0}=\left\{\mathrm{R}_{0}, \mathrm{U}_{-1}^{2}\right\}^{\prime \prime}$. Moreover we have the following decomposition for $s$ :

$$
s=\left(s \mathrm{Ad}_{0} \mathrm{U}_{-1}\right)\left(\mathrm{Ad} \mathrm{U}_{0}^{*} \mathrm{U}_{-1}^{*}\right)=s_{1} s_{2},
$$

where $s_{1}=s \mathrm{AdU}_{0} \mathrm{U}_{-1} \in \overline{\operatorname{Int}(\mathscr{B})}, \quad s_{2}=\operatorname{AdU}_{0}^{*} \mathrm{U}_{-1}^{*} \in \mathrm{Ct}(\mathscr{B})$. In addition $s_{i}^{2}=\operatorname{Ad} h$, with $h=\mathrm{U}_{0}^{2} g \in \mathscr{A}$ and $s_{i}(h)=-h$.

The decomposition above prevents $\mathscr{B}$ from being isomorphic to Jones's example ([8], in analogy with the construction in [4]). We recall that in Jones's example one considers $\mathrm{N}=\left(\mathscr{L}\left(\mathrm{F}_{\mathrm{N}}\right) \otimes \mathrm{R}_{0}\right) \rtimes_{\gamma} \mathbb{Z}_{2}$ for a suitable action $\gamma$. Then $\chi(\mathrm{N})=\mathbb{Z}_{2} \oplus \mathbb{Z}_{2}$, where, for exactly one of the two copies of $\mathbb{Z}_{2}$ in $\chi(\mathrm{N}) \subseteq \operatorname{Out}(\mathrm{N})$, there exists no obstruction for lifting to an action on N. By [4] (see also [8]) this copy (denoted by $\hat{\gamma}$ ) corresponds to the dual $\mathbb{Z}_{2}$ action for $N=\left(\mathscr{L}\left(F_{N}\right) \otimes R_{0}\right) \rtimes_{\gamma} \mathbb{Z}_{2}$. By Takesaki duality we get:

Proposition 7. - With the notations before, the dual action $\tilde{\gamma}$ on the cross product $\mathrm{N} \times \widehat{\gamma}_{\hat{\gamma}} \mathbb{Z}_{2}$, has decomposition of the form: $\tilde{\gamma}=t_{1} t_{2} \mathrm{AdW}$, where $t_{1} \in \mathrm{Ct}\left(\mathrm{N} \times \hat{\gamma}_{\hat{\gamma}}\right)$, $t_{2} \in \overline{\operatorname{Int}\left(\mathrm{N} \times{ }_{\hat{\gamma}} \mathbb{Z}_{2}\right)}$. Moreover there exists selfadjoint unitaries $e^{i}=e_{+}^{i}-e_{-}^{i}$, with $e_{ \pm}^{i}$ projections of trace $1 / 2$, so that $t_{i}^{2}=\mathrm{Ad} e^{i}, t_{i}\left(e^{i}\right)=-e^{i}, t_{i}\left(e_{j}\right)=e_{j}, i \neq j$.

CoRollary 8. - With the notations above, $\mathrm{N} \cong \mathscr{A}=\left(\mathscr{L}\left(\mathrm{F}_{k}\right) \otimes \mathrm{R}_{-1}\right) \star_{\mathrm{R}_{-1}} \mathrm{R}_{0}$.

Proof. - Assume the contrary. Then $\mathscr{B}=\mathscr{A} \times \mathbb{Z}_{2}, \mathrm{~N}=\left(\mathscr{L}\left(\mathrm{F}_{\mathrm{N}}\right) \otimes \mathrm{R}_{0}\right) \dot{\aleph}_{\gamma} \mathbb{Z}_{2}$ would be isomorphic (since in each of the two cases the $\mathbb{Z}_{2}$ action is canonical). Moreover it would follow that the corresponding dual actions have the same image in Out, and thus by [3], we may assume that they are equal.

Since in $N=\left(\mathscr{L}\left(\mathrm{F}_{\mathrm{N}}\right) \otimes \mathrm{R}_{0}\right) \times_{\gamma} \mathbb{Z}_{2}$, the decomposition of an automorphism as the product of a centrally trivial automorphism and an almost inner automorphism, is unique (modulo inner automorphism), it would follow that the decomposition $s=s_{1} s_{2}$ (regarded as an action of $\mathbb{Z}_{4} \oplus \mathbb{Z}_{4}$ ) is outer conjugate to the similar action described by $t_{1}$, $t_{2}$ in the preceding lemma. But this is impossible because the two actions have different characteristic invariants (see [9]). [Since $t_{1}\left(e_{2}\right)=e_{2},\left(t_{i}\right)^{2}=\mathrm{Ad} e^{i}$, while, with the notations in Proposition 5, $s_{i}(h)=-h, s_{i}^{2}=\mathrm{Ad} h$.]

Proposition 9. - Let $\mathrm{N}$ be the factor constructed in [8]. Then there exists subalgebras $\mathrm{A} \subseteq \mathrm{B}$ in $\mathrm{N}$ so that $\mathrm{A}$ is isomorphic to $\left(\mathscr{L}\left(\mathrm{F}_{k}\right), \mathrm{B}\right.$ is the cross product $\mathrm{A} \rtimes_{\theta} \mathbb{Z}_{4}$, where $\theta$ is $a \mathbb{Z}_{2}$-kernel on $\mathrm{A}$ with non-trivial obstruction (to lifting). 'Moreover $\mathrm{N}$ is the enveloping algebra for the inclusion $\mathrm{A} \leqq \mathrm{B}$ and $\mathrm{A}$ is isomorphic to $\mathscr{L}\left(\mathrm{F}_{k}\right)$.

Note remise le 10 février 1993, acceptée le 12 mars 1993.

\section{REFERENCES}

[1] M. CHODA and H. KosAd, Strongly outer actions for an inclusion of factors, Preprint, 1992.

[2] A. Connes, Periodic automorphisms of the hyperfinite $\mathrm{II}_{1}$ factor, Acta Sci. Math., 39, 1977, pp. 39-66.

[3] A. ConNes, Outer conjugacy classes of automorphisms of factors, Ann. Sci. École Norm. Sup., 8, fasc. 3,1975 . 
[4] A. Connes, Sur la clasșification des facteurs de type II, C. R. Acad. Sci., Paris, 281, Series A, 1975, pp. 13-15.

[5] A. Connes, A factor not anti-isomorphic to itself, Ann. of Math., 104, 1976, pp. 536-554.

[6] F. Goodman, P. DE LA HARpe and V. F. R. Jones, Coxeter graphs and towers of algebras, Coxeter graphs and towers of algebras, MSRI Publications 14, Springer Verlag, Berlin, Heidelberg, New York, 1989.

[7] V. F. R. Jones, Index for subfactors, Invent. Math., 72, 1983, pp. 1-25.

[8] V. F. R. JONES, A $\mathrm{II}_{1}$ factor anti-isomorphic to itself but without involutory antiautomorphisms, Math. Scand., 46, 1980, pp. 103-117.

[9] V. F. R. JoNES, Actions of finite groups, in Springer Lect. Notes in Math., 725, pp. 237-253.

[10] V. F. R. Jones, Baxterization, Intnl. J. Modem Phys. A, 6, No. 12, 1991, pp. 2035-2043.

[11] V. F. R. JoNES, Notes on Connes invariant $\chi(\mathrm{M})$, unpublished.

[12] V. F. R. Jones and C. E. SutHERLAND, unpublished manuscript.

[13] R. V. KaDISON, List of open problems at the Baton Rouge conference, 1967.

[14] Y. KaWAhIgashi, Centrally trivial automorphisms and an analogue of Connes's $\chi(\mathrm{M})$ for subfactors, Preprint, Berkeley, 1992.

[15] $\mathrm{Ph} . \mathrm{H}$. LoI, On the derived towers of certain inclusions of type $\mathrm{III}=\lambda$ factors of index 4 , Preprint.

[16] D. MCDuff, Central sequences and the hyperfinite factor, Proc. London Math. Soc., 21, 1976, pp. 443-461.

[17] A. Ocneanu, in Operator Algebras and Appl., 2, London Math. Soc. Lect. Notes, 136, 1988, pp. 202-236.

[18] A. OCNEANu, Actions of discrete amenable groups on von Neumain algebras, Springer Lect. Notes in Math., 1138, 1985.

[19] J. Phillups, Automorphisms of full $\mathrm{II}_{1}$ factors, Duke J. Math., 43, 1976, pp. 375-385.

[20] M. PIMSNer and S. PoPA, Entropy and index for subfactors, Amn. Sci. Ec. Norm. Sup., 19, 1986, pp. 57-106.

[21] S. PopA, Classification of subfactors: the finite depth case, Invent. Math., 101, 1990, pp. 19-43.

[22] S. POPA, Markov traces on universal Jones algebras and subfactors of finite index, Invent. Math.

[23] S. POPA, Classification of amenable subfactors of type II, IHES Preprint, No, 43, June 1992.

[24] F. RĂDULESCU, Random matrices, amalgameted free products and subfactors of the von Neumann algebra of a free group of non-integer index, Preprint IHES, 1991.

[25] E. STORMER, Conjugacy of Involutive Antiautomorphisms of von Neumann algebras, $J$. Funct. Analysis, 66, 1986, pp. 54-66.

[26] D. Volculescu, Circular and semicircular systems and free product factors, in Operator Alg., Unitary

Repr., Enveloping Algebras and Inv. Theory, Progress in Mathematics, 92, Birkhäuser, Boston, 1990.

[27] D. VorCulescu, in Operator Algebras and connections with Ergodic Theory, Springer Lecture Notes in Math., 1138, 1989.

[28] C. E. Sutherland, Cohomology and extensions of von Neumann algebras, Publ. RIMS, 16, 1980, pp. 105-133.

[29] M. TAKeSAKI, Duality and von Neumann Algebras, Springer Lecture Notes, No. 247.

Department of Mathematics, University of California at Berkeley, Berkeley, Ca 94720, USA and Institute of Mathematics of the Romanian Academy. 